\title{
Speeding Up Target Address Generation Using a Self-indexed FTB
}

\author{
Juan C. Moure, Dolores I. Rexachs, and Emilio Luque $e^{1}$ \\ Computer Architecture and Operating Systems Group, \\ Universidad Autónoma de Barcelona. 08193 Barcelona (Spain) \\ \{JuanCarlos.Moure, Dolores.Rexachs, Emilio.Luque\}@uab.es
}

\begin{abstract}
The fetch target buffer (FTB) holds information on basic blocks to predict taken branches in the fetch stream and also their target addresses. We propose a variation to FTB, the self-indexed FTB, which, through an extra level of indirection, provides the high hit rate of a relatively large, high-associative FTB with the fast access delay of a small, direct-mapped FTB. The critical and most frequent operation -predicting the next FTB entry- is speeded up, whilst less frequent operations -such as recovering from FTB misses- are slightly slowed down. The new design is both analyzed and simulated. Performance increase on a 512-entry FTB is estimated at between $15 \%$ and $30 \%$.
\end{abstract}

\section{Introduction}

The first stage on a processor's pipeline provides a stream of instruction addresses to the instruction cache (iCache). Since taken branches are detected later in the pipeline, they create holes in the instruction fetch sequence, and limit the processor's performance. The Branch Target Buffer (BTB) represents a hardware solution to the problem, and consists of storing previous history -branch addresses, target addresses, and previous branch behavior- to anticipate the occurrence of taken branches, [5,7]. Two kinds of prediction are carried out: target address generation -predicting the occurrence of taken branches and their target address- and branch prediction choosing the direction (taken or not) of conditional branches-. More flexibility is provided by using a dedicated structure for conditional branch prediction, distinct from the BTB [3]. This paper focuses exclusively on target address generation.

Address generation throughput and efficiency is increased by storing the size of each basic block (BB) -the group of sequential instructions starting with a target instruction and ending with a branch- within the target buffer [10]. Reinmann et al. proposed a multi-level, BB-based structure, called the Fetch Target Buffer (FTB), [8], which combines the low access delay of a small, first-level table with the high prediction accuracy of a large second-level table. Their approach is based on technological trends that seem to indicate that, as feature sizes shrink, circuit delays become increasingly limited by the amount of memory in the critical path, [14]. The

${ }^{1}$ This work was supported by the MCyT-Spain under contract TIC 2001-2592 and partially supported by the Generalitat de Catalunya - Grup Recerca Consolidat 2001 SGR-00218 
size and associativity of the L1 FTB determine its access delay, and cannot be overly reduced, or an excessive L1 miss rate will hamper overall FTB performance.

Following this approach, we propose the Self-indexed FTB (SiFTB), which reduces the average Level-1 FTB cycle time without increasing its miss rate. An additional table containing FTB indexes, rather than instruction addresses, provides an extra level of indirection that avoids costly associative searches on consecutive FTB hits. Associative searches are still required on FTB misses, but they should be infrequent. Section 2 provides fuller details on the FTB and SiFTB functionality.

Using CACTI, [9], we estimate lower and upper bounds for the SiFTB's delay advantage, ranging from between $30 \%$ to $70 \%$. Using simulation, we then calculate the frequency of hits and misses for each design. Combining delay bounds and simulation results, we found speedup to be very dependent on FTB size. For most useful sizes, speedup ranges from between $15 \%$ to $30 \%$. This is presented in section 3. Section 4 outlines the conclusions and sets out future lines of research.

\section{The FTB and the Self-indexed FTB}

In this section, we describe the FTB and SiFTB schemes and compare their advantages and disadvantages. We then discuss certain related issues.

Figure 1 depicts a simplified FTB. Each FTB's entry is tagged by the starting address of a $\mathrm{BB}$ (BBaddr field), and contains the type of the ending branch (type) as well as the addresses of the taken and not-taken BBs ( $t k B B$ and $n t B B)$, also called target and fall-through BBs. A separate Conditional Branch Predictor (CBP) is used, and a Return Address Stack (RAS) predicts the target of subroutine returns.

A Fetch Target Queue (FTQ) decouples the FTB and iCache stages, allowing each of these to be designed as a multi-cycle, pipelined hierarchy. It allows the FTB to run ahead of the iCache, and hides part of the FTB and iCache penalties, whilst its delay overhead is only exposed on mispredictions.

The current BB address is compared against the BBaddr tags every cycle. On a hit, the type value and $\mathrm{CBP}$ outcome select the next $\mathrm{BB}$ address from three options: $t k B B$ value (for unconditional jumps or predicted-taken conditional branches), $n t B B$ value (for predicted-not-taken conditional branches), and top of RAS (for return jumps). Call instructions also push the $n t B B$ value on the RAS. On a miss, a fixed constant $k$ is added to the current $\mathrm{BB}$ address to generate the next $\mathrm{BB}$ address.

When a BB not found in the FTB is identified in the decode stage, it is inserted into the FTB (LRU replacement). After a misprediction, the correct BB address is used on the FTB to resume target prediction. The CBP is updated as branches are solved.

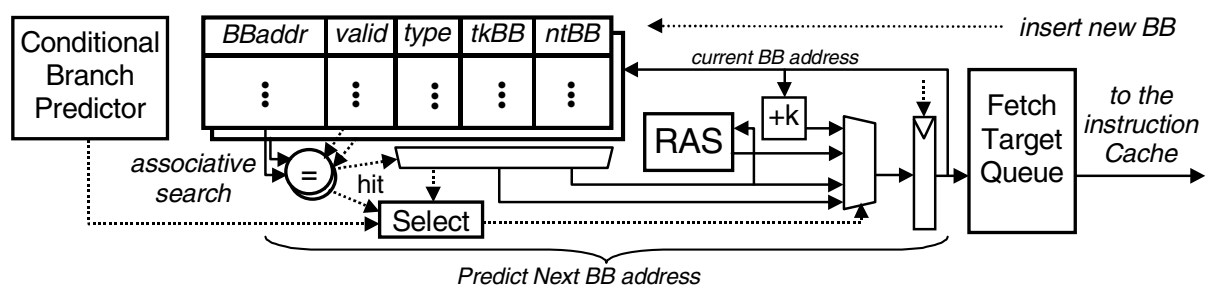

Fig. 1. Block diagram of a Fetch Target Buffer (FTB). 
The SiFTB includes a new self-indexed table (SiTable) containing the previous type field and two new index fields, $t k I d x$ and $n t I d x$, which are pointers to the SiFTB. A separate FTB array contains the rest of the fields (see Figure 2).

Every cycle, both tables are addressed in parallel using the current index: the SiTable provides the next SiFTB index, and the FTB array provides the next BB address. Predicting the next index is carried out as in the FTB. The type value and the CBP outcome select the next BB index from: $t k I d x$ value (unconditional or predictedtaken conditional), ntIdx value (predicted-not-taken conditional), and top of RAS (returns). It should be noticed that the RAS also contains indexes (calls push the ntIdx value on the RAS). The BB address corresponding to a SiFTB index is obtained one cycle before the index is obtained. At this time, target misses are checked by comparing the value of the BBaddr field to the previously predicted BB address.

$\mathrm{BBs}$ are inserted in the SiFTB when the index for the following BB is known, so that one of the index fields is correctly set, while the other index field initially points to a random entry within the set. If an index field points to an incorrect entry and the expected BB is stored in the other entry, the SiFTB may incur a false miss. This may also occur due to entry replacements.

On hits, the BB generation rate is speeded up because the SiTable is small (few and short fields) and direct-mapped (no associative comparison). SiFTB misses, however, do need associative search logic on the BBaddr fields to re-enter the chain of BB pointers. The associative search is avoided on conditional branch mispredictions by storing the index for the alternative path along with the predicted branch.

The SiFTB scheme is similar to a predictive set-associative cache (PSAC), [5 3.11]. The PSAC embeds a BTB into the iCache by using an index field to predict the next cache block set and way. It may incur way misses that are similar to those we call false misses. However, separating the FTB and the iCache, as in our proposal, allows for traversing full BBs instead of fixed-size cache blocks, thus maximizing instruction bandwidth per branch prediction, and permits the design of multi-cycle, pipelined iCaches and FTBs.

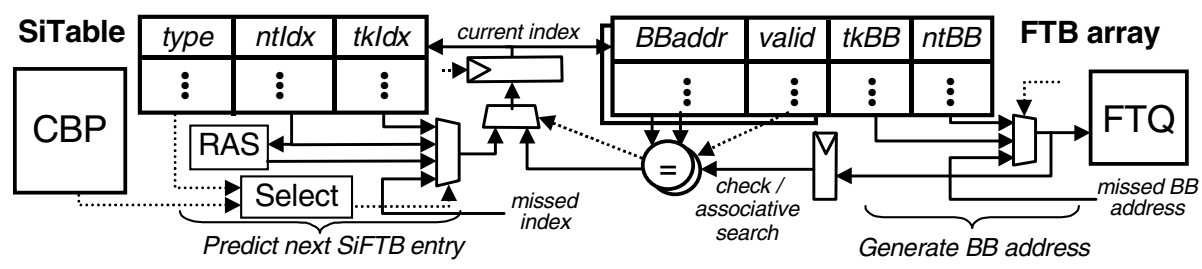

Fig. 2. Self-Indexed FTB: generating an index to the next SiFTB entry is decoupled from obtaining the BB address and checking SiFTB misses.

\section{Performance Evaluation}

We compare the performance of both designs by first estimating lower and upper bounds for the circuit delay differences on all possible execution cases; by using simulation, we then evaluate the frequency of these cases on several benchmarks.

The access time of a table for a varying number of entries (64 to $4 \mathrm{~K}$ ), entry sizes (14 bits to 70 bits), and associative degrees (direct-mapped and 4-way associative) 
was obtained using the CACTI cache simulator, [9]. The result is that a set-associative FTB is between $30 \%$ and $70 \%$ slower than a direct-mapped SiTable (with entries from 14 to 26 bits), and between $20 \%$ and $60 \%$ slower than a direct-mapped FTB array (with larger entries). On consecutive hits, then, the SiTable generates BBs that are between $30 \%$ to $70 \%$ faster than the FTB, which suffers the associative logic overhead. Storing the index for the alternative path on a conditional branch prediction avoids the overhead of the associative logic on a misprediction.

Recovering from a BB target miss always requires an associative search on both designs. Since the SiFTB is optimized for direct access, we assume that the delay for the associative access should be larger than on the FTB (between 5\% and 25\%). False misses on the SiFTB will therefore involve an extra penalty equal to the difference between a direct and an associative SiFTB access. We assume that the insertion of BBs into the FTB or SiFTB do not increase the miss penalty.

In our limit analysis, we assume the FTB/SiFTB is the only system bottleneck. Therefore, we only simulate hit/miss FTB/SiFTB behavior, ignoring time issues and conditional and indirect branch prediction, and assuming that tables are immediately updated. Because we assume that sequential instructions are injected by default in the case of misses, the penalty for recovering from a miss is only suffered after a taken branch, and many misses between two consecutive taken branches are only penalized as a single miss.

We have used release 3.0 of the SimpleScalar-Alpha simulation tools, [2], and the SPECint95 and SPECint00 benchmarks that impose more problems on the FTB/SiFTB (Figure 3.a). For each benchmark, we simulate the first thousand million instructions, and count the number of misses and false misses for a number of entries, $\boldsymbol{n}$, varying from 32 to 2048 . The proportion of false misses compared to true misses is small, but increases with the number of entries (from $2 \%$ to $10 \%$ of the total misses).

Figure 3.b shows the result of combining miss rates and cycle time speedup (Csp from 1.2 to 1.6 , considering values from the worst to the best case). When the number of entries is large enough to reduce misses bellow $1 \%$, overall performance speedup comes close to cycle time speedup, as expected. An excessive miss rate hampers the cycle time advantage of the SiFTB, as misses have a higher penalty on the SiFTB than on the FTB. Our selected benchmarks have large instruction working sets and require more than 512 entries to allow the $\mathrm{SiFTB}$ to achieve a total performance increase of $60 \%$ of cycle time speedup. With $C s p$ between 1.25 and 1.6, the average performance increase of the SiFTB versus the FTB will be $15 \%-30 \%$.

\begin{tabular}{ccc}
\hline benchmark & SPEC version & input data \\
\hline vpr & 2000 & reference \\
\hline gcc & 2000 & expr.i \\
\hline perlbmk & 2000 & splitmail \\
\hline crafty & 2000 & reference \\
\hline eon & 2000 & cook \\
\hline twolf & 2000 & reference \\
\hline vortex & 95 & reference \\
\hline li & 95 & reference \\
\hline go & 95 & reference
\end{tabular}

a) Benchmarks: DEC C compiler (-O4)

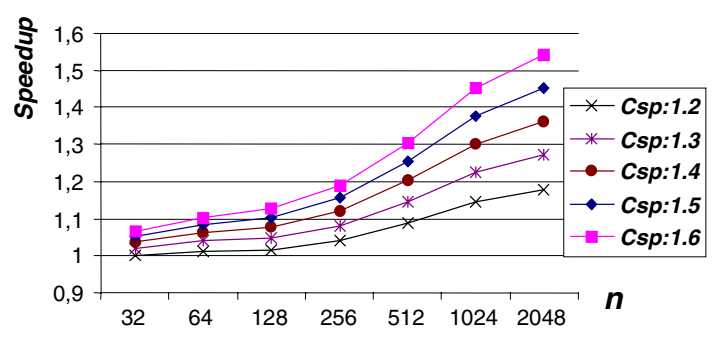

b) Performance Speedup of SiFTB versus FTB

Fig. 3. $C s p$ is cycle time speedup and $n$ is the number of FTB/SiFTB entries. Results are averaged for all the benchmarks. Placement is 4-way associative and replacement is LRU. 


\section{Conclusions and Future Work}

We have proposed a modification to the FTB, the Self-indexed FTB, which uses a small and direct-mapped table of SiFTB pointers to increase BB prediction rate on hits. Misses, however, have a slightly larger penalty and new false misses may occur. Using simulation, we have calculated the frequency of misses generated by the FTB and the SiFTB and found a small proportion of false misses. Nevertheless, the SiFTB's delay advantage is only exploited when the number of total misses is small, i.e., for tables with a relatively large number of entries.

In order to exploit all the proposal's possibilities, other bottlenecks should be removed. For example, conditional branch mispredictions will waste a substantial part of the SiFTB advantage. We have also ignored the fact that a very fast SiFTB requires a very fast CBP. Reinmann et al. embedded the CBP into the FTB, [8], and there are techniques, such as the lookahead prediction, [10], and the overridden prediction, [4] to avoid branch prediction from becoming a cycle-time bottleneck.

There are many FTB variations that we have not analyzed in this paper. Reinmann et al. considered dynamic BBs that may embed several not-taken conditional branches, [8], and analyze a two-level FTB hierarchy. We will consider these issues in the future. Since a single SiFTB suffers if the number of entries is reduced, adding an extra L2 SiFTB may reduce the penalty of L1 misses and extend the delay advantage on hits to very small L1 SiFTBs.

\section{References}

1. V.Agarwal, M.S.Hrishikesh, S.W.Keckler, D.Burger: Clock Rate versus IPC: The End of the Road for Conventional Microarchitectures. Proc. ISCA-27, (2000) 248-259

2. D.Burger, T.M.Austin: The SimpleScalar Tool Set. Univ. Wisconsin-Madison Computer Science Department, Technical Report TR-1342, 1997

3. B.Calder, D.Grunwald: Next Cache Line and Set Prediction. Proc. ISCA-22, (1995) 287296

4. D.A. Jimenez, S.W. Keckler, C. Lin: The Impact of Delay on the Design of Branch Predictors. Proc. MICRO-33, (2000) 67-76

5. M.Johnson: Superscalar Microprocessor Design. Innovative Technology. Prentice-Hall Inc., Englewood Cliffs, NJ, (1991)

6. J.K.F.Lee, A.J.Smith: Branch Prediction Strategies and Branch Target Buffer Design. IEEE Computer (1984) 17(2): 6-22

7. C.H.Perleberg, A.J.Smith: Branch Target Buffer Design and Optimization. IEEE Trans. on Computers (1993) 42(4): 396-412

8. G.Reinman, T.Austin, B.Calder: A Scalable Front-End Architecture for Fast Instruction Delivery. Proc. ISCA-26, (1999) 234-245

9. G.Reinman, N.Jouppi: An Integrated Cache Timing and Power Model. COMPAQ Western Research Lab, http://www.research.digital.com/wrl/people/jouppi/CACTI.html (1999)

10. T.-Y.Yeh, Y.N.Patt: A Comprehensive Instruction Fetch Mechanism for a Processor Supporting Speculative Execution. Proc. MICRO-25, (1992) 129-139

11. R. Yung: Design Decisions Influencing the Ultrasparc's Instruction Fetch Architecture. Proc. MICRO-29, (1996) 178-190 